\title{
Postoperative metastasis prediction based on portal vein circulating tumor cells detected by flow cytometry in periampullary or pancreatic
} cancer

This article was published in the following Dove Press journal:

Cancer Management and Research

\section{Lianyuan Tao ${ }^{1,2}$ \\ $\mathrm{Li} \mathrm{Su}{ }^{3}$ \\ Chunhui Yuan' \\ Zhaolai Ma' \\ Lingfu Zhang' \\ Shiping $\mathrm{Bo}^{4}$ \\ Yunyun $\mathrm{Niu}^{4}$ \\ Sijia Lu ${ }^{4}$ \\ Dianrong Xiu'}

'Department of General Surgery, Peking University Third Hospital, Beijing 100191,

People's Republic of China; ${ }^{2}$ Department of Hepatobiliary Surgery, Henan

Provincial People's Hospital, People's

Hospital of Zhengzhou University, School

of Clinical Medicine, Henan University,

Zhengzhou, Henan 450003, People's

Republic of China; ${ }^{3}$ Medical and Health

Analytical Center, Peking University

Health Science Center, Beijing I00191,

People's Republic of China; ${ }^{4}$ Department of Clinical Research, Yikon Genomics Co. Ltd., Shanghai, People's Republic of China

Correspondence: Dianrong Xiu Department of General Surgery, Peking University Third Hospital, No. 49, Hua

Yuan North Road, Hai Dian District,

Beijing 100191, People's Republic of China

Tel +86 I08 2267320

Fax +86 I06 20I 0334

Email xiudianrong@hotmail.com
Purpose: The aim of this study was to evaluate the value of flow cytometry (FCM) detection of portal vein circulating tumor cells (CTCs) in predicting postoperative metastasis. Methods: Samples of portal venous blood and peripheral blood were collected from 39 patients during surgery, and CTCs were detected by FCM, with confirmation by laser confocal microscopy and single-cell sequencing.

Results: Among all patients, a portal EpCAM+CD45- percentage $\geq 24.5 \times 10^{-4}(P=0.06)$, peripheral EpCAM+CD45- count $\geq 97 / 5 \mathrm{~mL}(P=0.034)$, peripheral EpCAM+CD45- percentage $\geq 4.4 \times 10^{-4}(P=0.042)$, and $\mathrm{CA} 242 \geq 3.5 \mathrm{U} / \mathrm{mL} \quad(P=0.027)$ were significant predictors of metastasis. Further analysis showed that the portal EpCAM+CD45- ratio $\geq 24.5 \times 10^{-4}$ is a predictor of metastasis $(P=0.025)$ in pancreatic cancer after curative resection.

Conclusion: CTCs detected by FCM in portal venous blood are of significant value for the prediction of postoperative metastasis in pancreatic or periampullary tumors.

Keywords: circulating tumor cells, flow cytometry, portal vein, pancreatic cancer, metastases

\section{Background}

Pancreatic and periampullary (located near the ampulla of Vater) cancers are lethal diseases with a very poor prognosis. ${ }^{1-4}$ The 5-year overall survival (OS) of pancreatic cancer (PC) patients is $3-7 \%$, whereas that of periampullary cancer patients is at $23-32.8 \% .^{2-6}$ We previously reported that approximately $70 \%$ of PC patients die of distant metastasis after curative surgery (84.3\% with liver metastasis) ${ }^{7}$ and a similar trend is also observed in periampullary cancer. ${ }^{8}$ Therefore, as metastasis is the main reason for the poor postoperative prognosis of pancreatic and periampullary cancers, it is vital to establish additional prognostic markers for metastasis.

Many studies have shown that circulating tumor cells (CTCs) play important roles in metastasis, and this is gradually becoming a key focus of cancer research. ${ }^{9,10}$ One meta-analysis indicated that the peripheral blood CTC count can be an informative biomarker for the prognostic evaluation of PC patients. ${ }^{11}$ However, this finding may be significantly limited in clinical applications. Indeed, peripheral venous blood tumor cell detection in digestive system tumors lacks sensitivity due to the low number of cells. Although studies have shown that CTCs in portal venous blood collected during surgery might be related to 
postoperative liver metastasis, ${ }^{12-16}$ these studies have several limitations that hinder their clinical application. First, application of microfluidic chips lacks validation, ${ }^{13}$ and the data collected are limited and lack statistical significance due to the small sample size. ${ }^{12}$ Second, the results obtained through the platforms used in the above two studies were unstable. the main current platforms for CTC detection are the CellSearch system, ${ }^{12,15,17}$ microfluidic capture ${ }^{13,14,18}$ and size-based filtration, ${ }^{16,19}$ which detect the number of CTCs in a certain volume of blood. However, the number of CTCs may be not stable during surgery due to the large volume of blood loss or fluid infusion, which may significantly disturb the stability of the CTC count. Lastly, these platforms are either too complex or expensive, and some of them require special equipment. All of these factors may preclude their utilization in clinical settings.

Recently, flow cytometry (FCM), a technique widely used in the clinic, has been suggested as an effective approach with high sensitivity and specificity for detecting and isolating CTCs. ${ }^{20} \mathrm{FCM}$ not only displays the number of CTCs detected but also determines the percentage of CTCs among certain subtypes of blood cells, which may prevent bias from variations in blood counts. However, there is still no report evaluating the effectiveness of FCM as a method of CTC detection and isolation in clinical settings. Therefore, in this study, we aimed to evaluate the value of CTC detection by FCM in both portal and peripheral venous blood collected during surgery in predicting postoperative metastases in pancreatic and periampullary tumors, with a particular focus on peripheral venous blood in PC.

\section{Materials and methods}

\section{Ethics statement}

The study was approved by the Clinical Ethics Committee of Peking University Third Hospital. Written informed consent was obtained from each patient. This study was conducted in accordance with the Declaration of Helsinki. All data were analyzed anonymously. All procedures were performed in accordance with relevant guidelines and regulations.

\section{Study population and design}

All participants were enrolled from Peking University Third Hospital (Beijing, China) between January 2016 and January 2017. Pancreatic or periampullary tumor patients who underwent curative resection via laparotomy and whose diagnoses were confirmed by pathological examination were included in the study. None of the patients had begun definitive tumor-specific treatment before blood sampling. All surgical specimens were evaluated pathologically to determine the extent of tumor differentiation, lymph node metastasis, and surgical margins following surgery. Pathological staging was determined according to the American Joint Committee on Cancer (AJCC) 7th Edition. ${ }^{21}$

\section{Blood sampling, safety, and feasibility}

During laparotomy, we first inspected and palpated the liver and examined the peritoneal cavity to identify any possible metastases. The portal vein was exposed after isolation and division of the common bile duct to its junction with the cystic duct. Five milliliters of blood was then collected from the portal vein via direct puncture using a 23-gauge needle before manipulation of the tumor; $5 \mathrm{~mL}$ of peripheral venous blood was also collected at the same time. The blood samples were transferred to $5-\mathrm{mL}$ ethylenediaminetetraacetic acid vacutainer tubes (BD, $\mathrm{K} 2 \mathrm{E}$ ), stored at $4-8{ }^{\circ} \mathrm{C}$ and processed within $6 \mathrm{hrs}$ of collection.

\section{Sample processing and CTC detection}

CTCs were detected by FCM (Gallios, Beckman Coulter, American) after collection of the mononuclear layer of cells. Briefly, blood mononuclear cells (BMCs) and CTCs were isolated by density gradient centrifugation with separation media (HLSM1077, Multi Sciences) according to the product instructions. The cells were washed twice with phosphate-buffered saline (PBS) and either used immediately for FCM detection with EpCAMallophycocyanin (APC) (cat: 324,208; BioLegend) (1:50) and CD45-Alexa Fluor $^{\circledR} 488$ (cat: ab187577; Abcam) (1:50) or stored in cell-freezing medium at $-80{ }^{\circ} \mathrm{C}$. The stored cell samples underwent flow cytometry within 14 days. $^{22}$

\section{Validation of FCM for CTC detection}

The recovery, sensitivity, linearity, specificity, and robustness of FCM for the detection of CTCs in colorectal carcinoma cancer have been previously evaluated. ${ }^{20}$ To verify the reliability of FCM for detection of CTCs in PC, we first validated the method using laser confocal microscopy, followed by single-cell sequencing of the detected cells. Possible tumor cells in specific FCM regions were randomly isolated under a microscope through manual separation of individual cells by 
micropipetting from one specimen, and whole-genome amplification was performed to detect copy number variation (CNV) profiles.

DNA was extracted from a single cell and subjected to next-generation sequencing (NGS) library preparation using the MALBAC Kit (Yikon Genomics). Sequencing was performed using an Illumina HiSeq 2500 sequencer, and approximately $1.5 \mathrm{M}$ sequencing reads were obtained from each sample. Chromosomal CNV analysis was performed following a previously described procedure. ${ }^{23}$ In brief, after removal of the Illumina adaptors and lowquality bases, high-quality reads were mapped to the hg19 reference genome using BWA (Burrows-Wheeler Alignment Tool, version 0.7.12-r1039) with default parameters. Uniquely mapped reads were extracted from the alignment reads. The entire reference genome was divided into non-overlapping observation windows (bins) with a size of $1 \mathrm{Mb}$, and the read number and GC content were calculated for each bin. To remove bias, the bin read count was normalized based on GC content and a reference dataset to determine the relative copy number. A copy number increase from two to three copies results in a 50\% increase in read count in a particular genome segment, and a copy number decrease from two to one results in a $50 \%$ decrease in read count in a genome segment.

To ensure that the cancer-specific CNV profiles were specific for CTCs in PC, we further assessed the CNV profiles of CTCs, which were confirmed by both immunofluorescence and morphology. Immunofluorescence detection was performed using tumor markers (Hoechst, CD45-PE and EpCAM-fluorescein isothiocyanate (FITC)). The criteria for CTC determination based on morphology were the same as those described in our previous study. ${ }^{7}$ Individual CTCs were isolated under a fluorescence microscope after manually separating individual cells by micropipetting from two specimens. Selected cells were processed for single-cell wholegenome amplification, and copy number variant regions were identified according to a previously described procedure. $^{24,25}$

\section{Collection of ctcs detected by FCM}

Two types of FCM parameters for CTC detection were analyzed. The number of EpCAM+CD45- cells detected in $5 \mathrm{~mL}$ of blood and the EpCAM+CD45- cell count were recorded. The percentage of EpCAM+CD45- cells among all BMCs was recorded as the EpCAM+CD45- percentage; using this percentage avoids biased measurements that can occur with variations in blood counts.

\section{Postoperative follow-up}

Patients were followed up postoperatively every 3 months for 1 year. During follow-up, they underwent a physical examination, laboratory examinations including measurement of tumor markers (CA19-9), chest radiography, abdominal ultrasound and abdominal magnetic resonance imaging (MRI) or computed tomography (CT). If newly developed lesions were found through CT scanning or ultrasound, with no definite evidence of metastasis from other cancers or recurrence elsewhere, we considered these to be metastases, and some were characterized by positron emission tomography (PET)-CT. Due to the possibility of seeding tumor cells via the needle tract, metastasis was not routinely confirmed by needle biopsy. The patients were followed up until death or December 31, 2017. Time to metastasis was measured from the day of surgery to the date of the diagnosis of metastasis. Overall survival time was measured from the day of surgery to the date of death or the last follow-up.

\section{Statistical analysis}

Mann-Whitney U tests were used to describe the normality of each continuous parameter's distribution, and $\chi^{2}$ tests were used for analysis of categorical variables. Quantitative results are reported in the form of means \pm standard deviation. Associations between clinical or histopathological parameters and metastasis, disease-free survival (DFS) or overall survival (OS) were analyzed. Univariate Cox regression analyses were performed to determine the effects of possible prognostic factors. Survival curves were constructed using the Kaplan-Meier method and compared with log-rank tests. Hazard ratios (HRs) for each independent variable were estimated from Cox analysis, and the results are shown as relative risks with corresponding 95\% confidence intervals (CIs). All the above analyses were performed using SPSS 22.0 statistical software (SPSS, IL, USA). $P<0.05$ was considered statistically significant. The recommend cutoff points of FCM parameters in the prognosis analysis were identified using selected log-rank statistics in R v. 3.0.0 (http://www.r-project. org/), as described in a previous study. ${ }^{26}$ We then selected the best cutoff point according to the participant distribution into two groups for all patients as well as for PC patients only. 


\section{Results}

Patient characteristics, safety, and feasibility Thirty-nine patients with a mean age of 63.0 years (SD, $\pm 8.7 \mathrm{y}$; range, 44-84 y) were enrolled, including 20 men (51.3\%) and 19 women (48.7\%). Further patient characteristics, clinical staging, and tissue sample findings are summarized in Table S1. Twenty-four patients were confirmed to have PC; among the remaining patients, 8 were diagnosed with ampullary adenocarcinoma, 5 with duodenal papillary adenocarcinoma, and 2 with cholangiocarcinoma. No immediate or delayed complications, including hematoma formation or gastrointestinal bleeding, were observed after portal vein sampling. Two peripheral blood specimens, one from a PC patient and another from a duodenal papillary carcinoma patient, failed the detection procedure due to coagulation and were not included in the study.

\section{CTC detection by flow cytometry and method validation}

Mononuclear cells were first isolated by density gradient centrifugation (Figure 1A). After separating possible cell debris, we performed further analysis on the cell region by flow cytometry, with cells in the EpCAM+CD45region considered to be tumor cells (Figure 1B). To enhance the practical relevance of this method, cells from different regions were identified by laser confocal microscopy (Figure 1C). Compared to EpCAM-CD45+ white blood cells (WBCs), cells in the EpCAM+CD45region exhibited the classical characteristics of CTCs. EpCAM + CD45+ cells are shown in Figure 1D in two forms $($ EpCAM $+\mathrm{CD} 45+(1)$ and $\mathrm{EpCAM}+\mathrm{CD} 45+(2))$; the atypical characteristics can be recognized as the combination of CTCs and WBCs (EpCAM+CD45+ (1)) or some other cell type that cannot be verified (EpCAM $+\mathrm{CD} 45+(2))$.

The CNV profiles of single randomly isolated individual EpCAM+CD45- or EpCAM+CD45- cells are shown in Figure 1C. With regard to EpCAM+CD45- cells, four (EpCAM $+\mathrm{CD} 45-\# 1, \# 3$, \#4 and \#5) were found to be tumor cells, and the remaining cell (EpCAM+CD45- \#2) was a WBC. Two of the cells in the EpCAM+CD4+ region (EpCAM+CD45+ \#1 and $\# 2$ ) were WBCs, and we were unable to identify the other two (EpCAM+CD45+\#3 and \#4) (Table S2). Therefore, cells in the $\mathrm{EpCAM}+\mathrm{CD} 45$ - region were identified as CTCs. FCM parameters including the EpCAM+CD45- cell count (CTCs per $5 \mathrm{~mL}$ of blood) and the EpCAM+CD45- percentage (percentage of CTCs among total BMCs) were collected for further analysis.

Further single-cell CNV detection of CTCs and WBCs in two samples selected under immunofluorescence and morphology analysis were performed. The determination of CTCs was based on EpCAM+ or CD45- profile and significant tumor cell morphology (Figure S1). Three identified CTCs and three WBCs were selected from each sample. The results showed that the CNV patterns for each CTC were distinctly different from those of normal single WBCs (Figure S2). The identified CTCs in the two samples (CTC \#1, \#2, \#3, \#4, \#5 and \#6) showed CNV patterns similar to those of the above CTCs (EpCAM $+\mathrm{CD} 45-\# 1, \# 3$, \#4 and \#5) isolated by FCM. Therefore, our results indicate that FCM may also be a reliable method for CTC detection and isolation in clinical settings.

\section{Prognostic value of clinicopathological variables and CTC count in all patients}

Comparison between peripheral and portal EpCAM + CD45- CTCs shows that both the EpCAM+CD45- percentage $\left(662.89 \pm 1143.54\right.$ vs $418.95 \pm 696.88, \quad\left(\times 10^{4}\right)$, $P=0.016)$ and EpCAM+CD45- count $(38.37 \pm 89.46$ vs $21.47 \pm 51.16,(/ 5 \mathrm{~mL}), P=0.024)$ were higher in portal venous blood than in peripheral venous blood.

We first analyzed the relationship between FCM parameters in all patients DFS as a continuous variable and found four FCM parameters to be associated with DFS: portal EpCAM+CD45- count, $P=0.024$ for cutoff $59 / 5 \mathrm{~mL}$; portal EpCAM $+\mathrm{CD} 45$ - percentage, $P=0.018$ for cutoff $4.2 \times 10^{-4}$; peripheral EpCAM $+\mathrm{CD} 45$ - count, $P=0.03$ for cutoff $96 / 5 \mathrm{~mL}$; and peripheral EpCAM+CD45- percentage, $P=0.015$ for cutoff $12.86 \times 10^{-4}$. The cutoff points were then adjusted based on the participant distribution for all patients as well as for PC patients only, to $60 / 5 \mathrm{~mL}$ and $24.5 \times 10^{-4}$ for the former and $97 / 5 \mathrm{~mL}$ and $4.4 \times 10^{-4}$ for the latter (Figure S3).

We performed the prognostic analysis using the above cutoff values. Our results indicated that a portal EpCAM $+\mathrm{CD} 45$ - count $\geq 60 / 5 \mathrm{~mL}$ (HR=8.644, 95\% CI $(1.133-$ 65.925); $P=0.037)$, a portal EpCAM+CD45- percentage $\geq 24.5 \times 10^{-4} \quad(\mathrm{HR}=3.447, \quad 95 \% \quad$ CI $\quad(1.225-9.705)$; $P=0.019)$, a peripheral EpCAM $+\mathrm{CD} 45$ - count $\geq 97 / 5 \mathrm{~mL}$ $(\mathrm{HR}=7.851, \quad 95 \% \quad$ CI $\quad(1.747-35.286) ; \quad P=0.007)$, a peripheral EpCAM+CD45- percentage $\geq 4.4 \times 10^{-4}$ $(\mathrm{HR}=7.204, \quad 95 \% \quad \mathrm{CI} \quad 1.609-32.256 ; \quad P=0.01), \quad$ and 
A

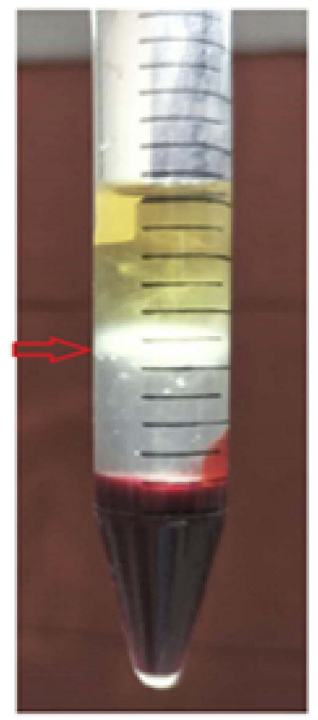

B

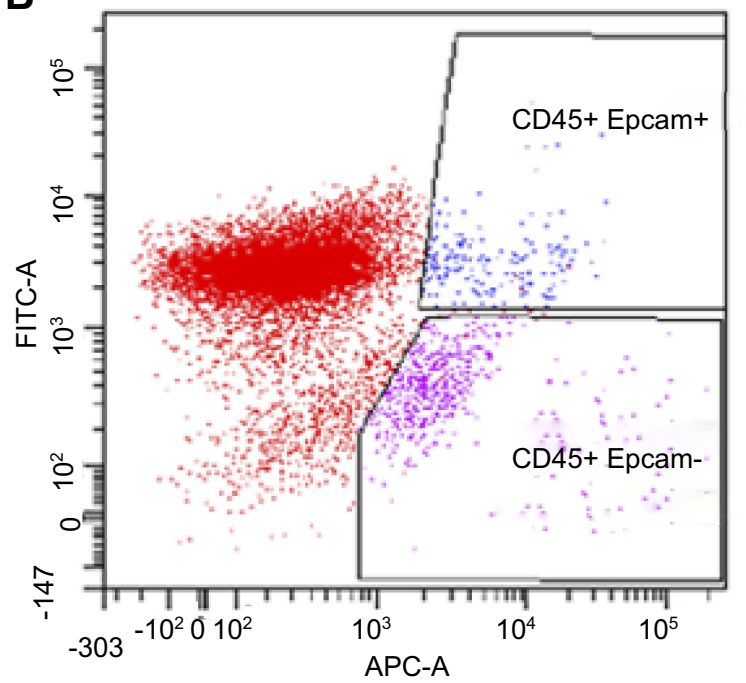

C

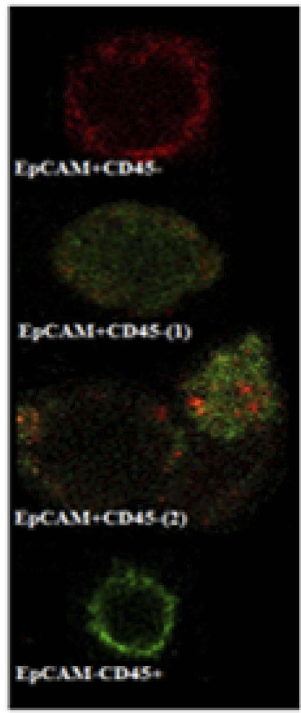

D
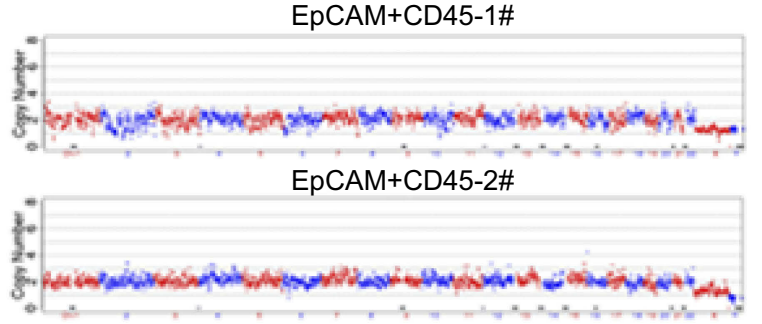

EpCAM+CD45-3\#

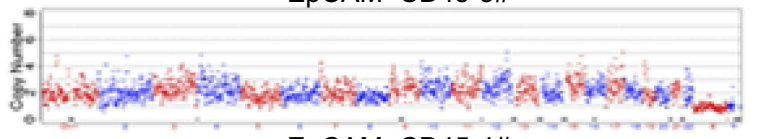

EpCAM+CD45-4\#

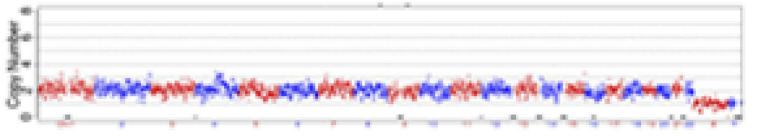

EpCAM+CD45-5\#

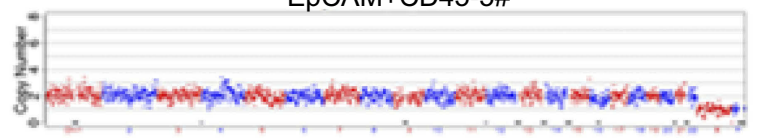

EpCAM-CD45+1\#

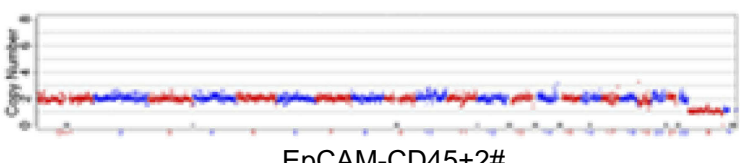

EpCAM-CD45+2\#

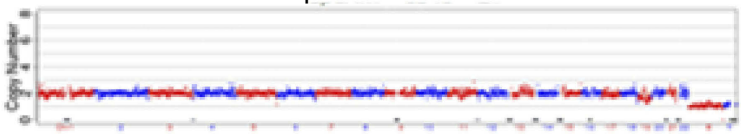

EpCAM+CD45+3\#

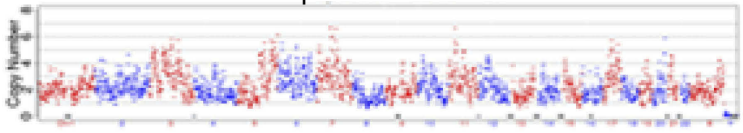

EpCAM+CD45+4\#

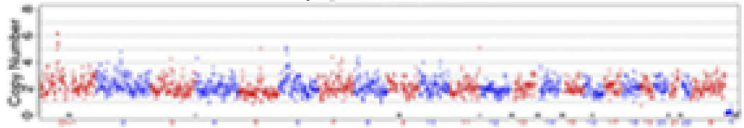

Figure I Sample processing and CTC detection. Blood mononuclear cells (BMCs) and CTCs were isolated by density gradient centrifugation (A). EpCAM+ and CD45- cells identified by flow cytometry were considered tumor cells (B). Cells from different regions were identified by laser confocal microscopy (EpCAM-APC: Red: CD45-Alexa Fluor $^{\circledR}$ 488: Green) (C). Cells randomly isolated by flow cytometry from the EpCAM+ CD45- region and the EpCAM+CD45+ region of one specimen were manually separated by micropipetting under a microscope, after which individual cells were subjected to whole-genome amplification to detect copy number variation (CNV) profiles. The copy numbers are segmented (blue and red lines) (D).

$\mathrm{CA} 242 \geq 3.5 \mathrm{U} / \mathrm{mL} \quad(\mathrm{HR}=6.937,95 \%$ CI $1.893-25.423$; $P=0.003$ ) were significantly associated with DFS in all patients. Further analysis showed that a portal EpCAM $+\mathrm{CD} 45$ - percentage $\geq 24.5 \times 10^{-4} \quad(\mathrm{HR}=3.015$, 95\% CI 0.954-9.53; $P=0.06)$, a peripheral EpCAM+CD45- count $\geq 97 / 5 \mathrm{~mL}(\mathrm{HR}=5.278,95 \%$ CI 1.132-24.613; $P=0.034)$, a peripheral EpCAM+CD45- percentage $\geq 4.4 \times 10-4$ $(\mathrm{HR}=4.931,95 \% \quad \mathrm{CI} \quad 1.063-22.863 ; \quad P=0.042)$, and $\mathrm{CA} 242 \geq 3.5 \mathrm{U} / \mathrm{mL} \quad(\mathrm{HR}=4.515,95 \%$ CI $1.183-17.234$;
$P=0.027$ ) were significant predictors of metastasis in these patients (Table 1). Kaplan-Meier curve analysis of metastasis and DFS in all patients (periampullary and pancreatic tumors) based on a portal EpCAM+CD45percentage $\geq 24.5 \times 10-4$ vs $<24.5 \times 10^{-4}$, as well as on a portal EpCAM+CD45- count $\geq 60 / 5 \mathrm{~mL}$ vs $<60 / 5 \mathrm{~mL}$, are shown in Figure 2. No statistically significant results associated with OS were found among the overall patient group or the PC patient subset. 


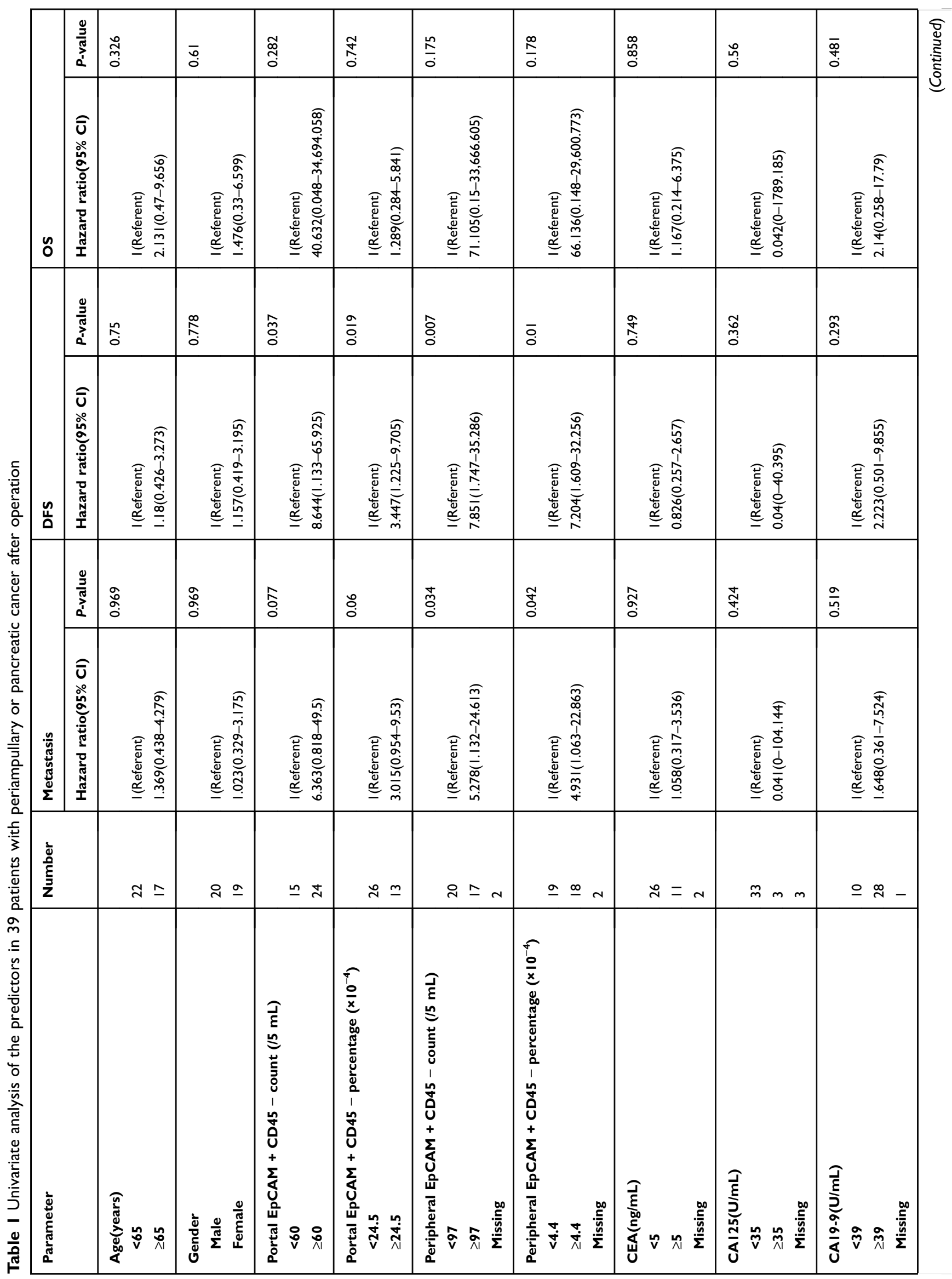




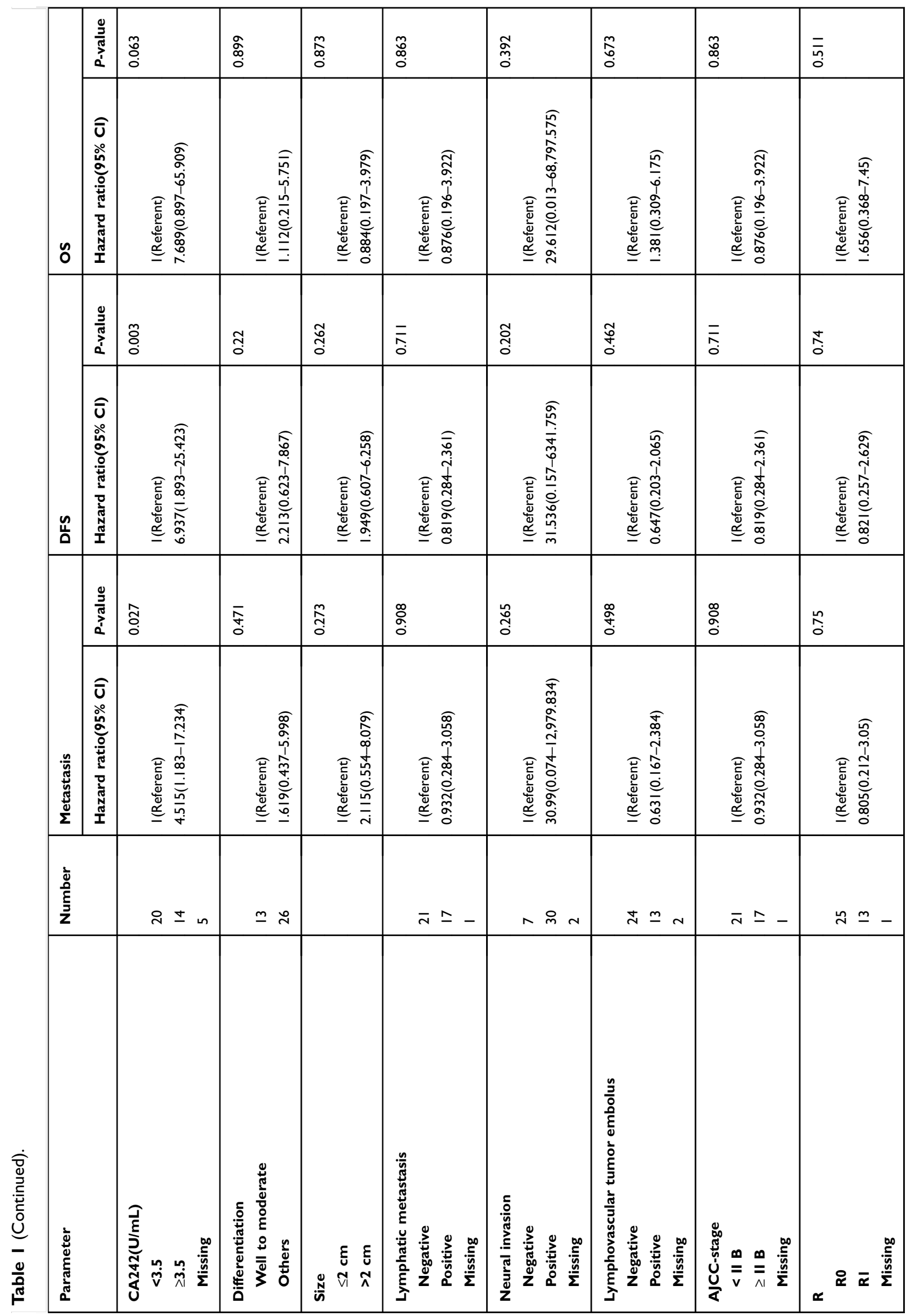



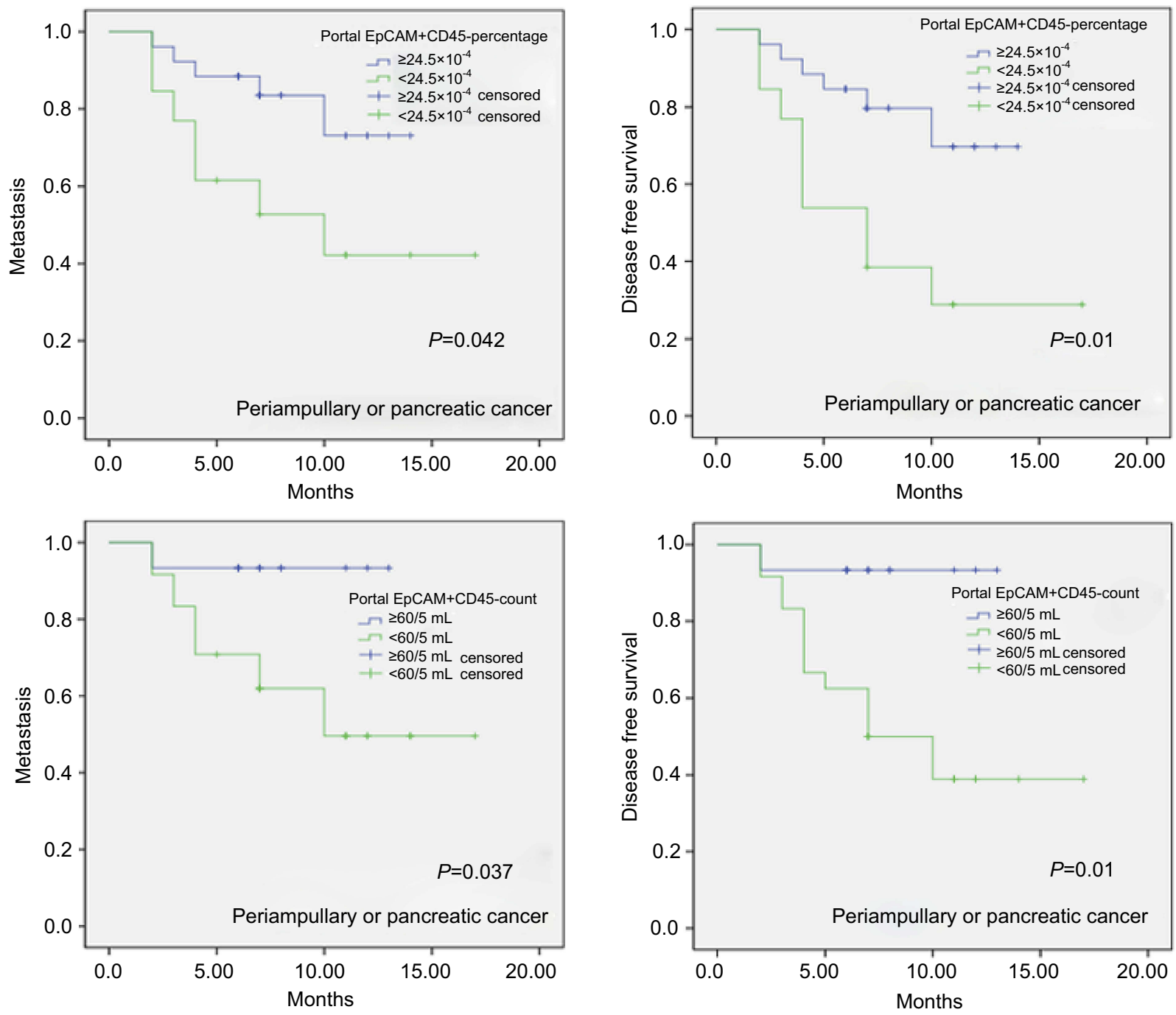

Figure 2 Kaplan-Meier curve of metastasis and disease-free survival in all patients (pancreatic and periampullary tumors) based on a portal EpCAM+CD45- percentage cutoff of $\geq 24.5 \times 10^{-4} \mathrm{vs}<24.5 \times 10^{-4}$ and a portal EpCAM+CD 45 - count cutoff of $\geq 60 / 5 \mathrm{~mL}$ vs $<60 / 5 \mathrm{~mL}$.

\section{Prognostic value of clinicopathological variables and CTC count in pancreatic cancer patients}

Based on the above cutoff values for FCM parameters, our results showed that a portal EpCAM+CD45- percentage $\geq 24.5 \times 10^{-4}$ is a significant predictor of both DFS $(\mathrm{HR}=5.525,95 \%$ CI 1.183-25.802; $P=0.03)$ and metastasis $(\mathrm{HR}=10.757,95 \%$ CI $1.354-85.446 ; P=0.025)$ after curative resection in PC patients (Table 2). Kaplan-Meier curve analyses of metastasis and DFS in PC patients based on a portal EpCAM+CD45- percentage cutoff of $\geq 24.5 \times 10^{-4}$ vs $<24.5 \times 10^{-4}$ and a portal EpCAM+CD45count cutoff of $\geq 60 / 5 \mathrm{~mL}$ vs $<60 / 5 \mathrm{~mL}$ are shown in Figure 3.

\section{Discussion}

The results of this study show that collection of portal venous blood during surgery for CTC enumeration by FCM is a feasible, safe and valuable approach for metastasis prognosis in patients with periampullary or pancreatic tumors. FCM has already been proven to be an efficient, inexpensive and easy platform for CTC detection. ${ }^{20}$ In the present study, we verified FCM-detected EpCAM+CD45cells using laser confocal scanning microscopy and singlecell sequencing. Moreover, cancer-specific CNVs have been confirmed to offer potential for CTC-based cancer diagnostics in lung cancer, ${ }^{25}$ and this approach has been recommended for CTC identification. ${ }^{24}$ In our study, CNV profiles of single cells randomly isolated from the FCM 


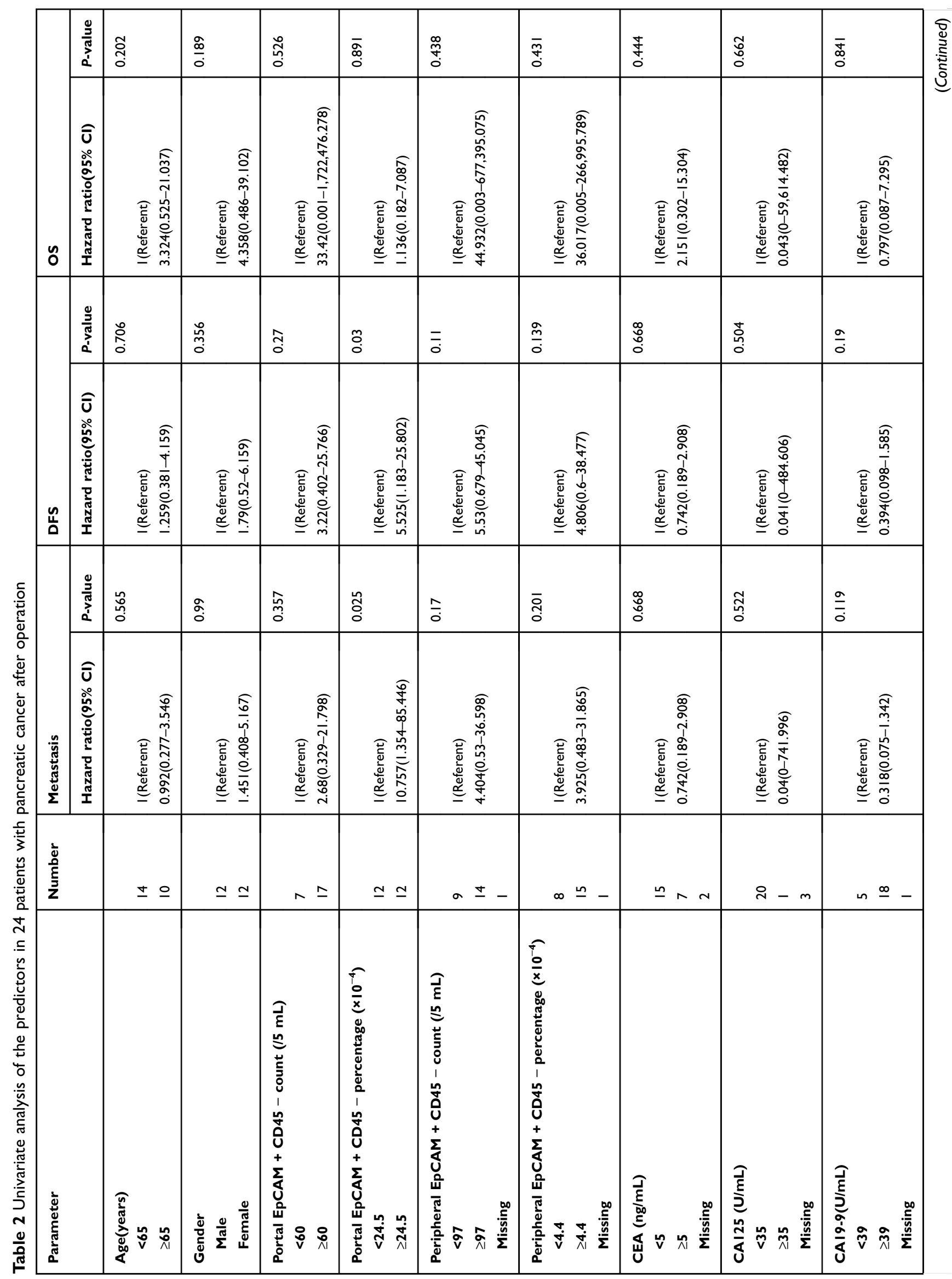




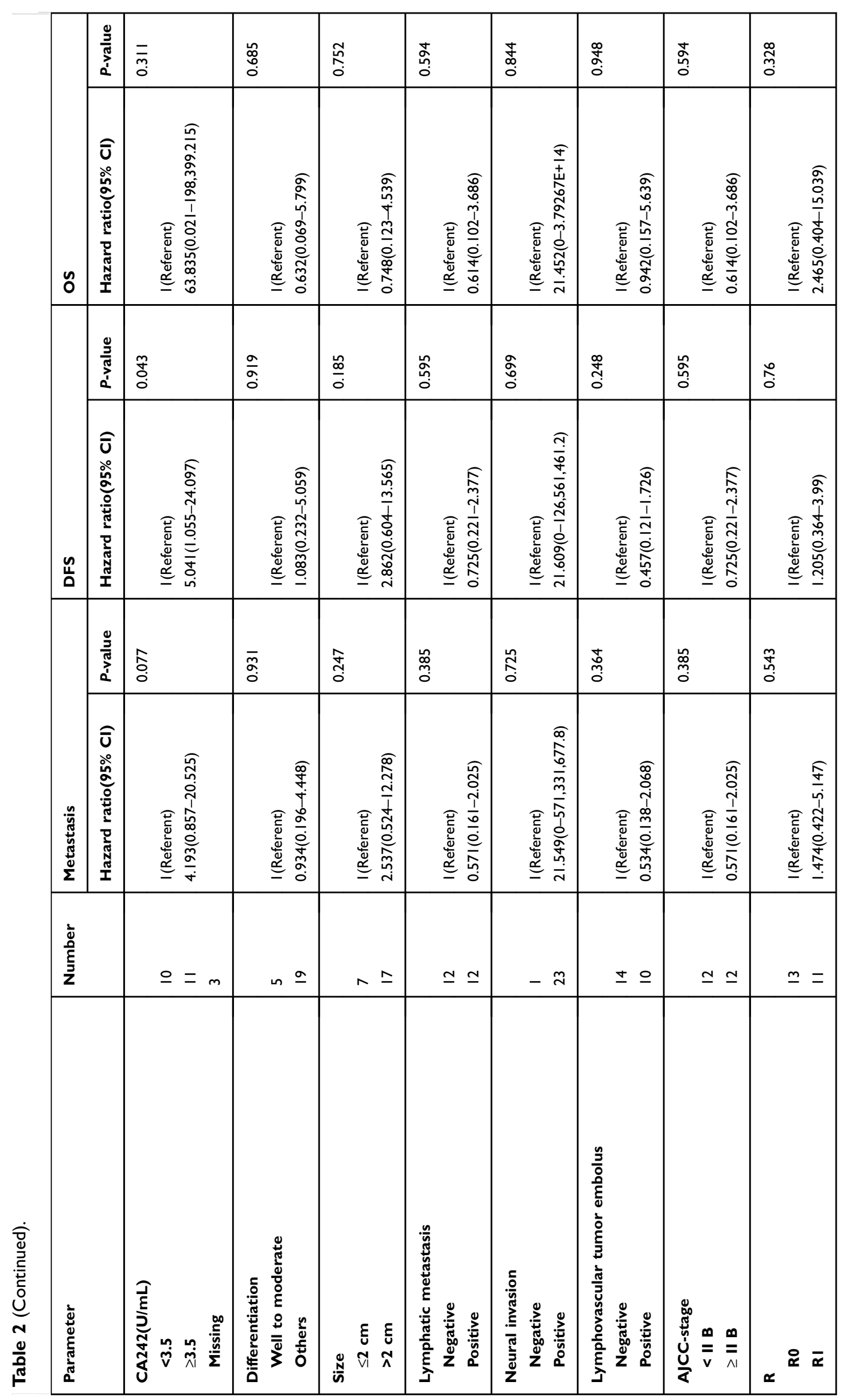



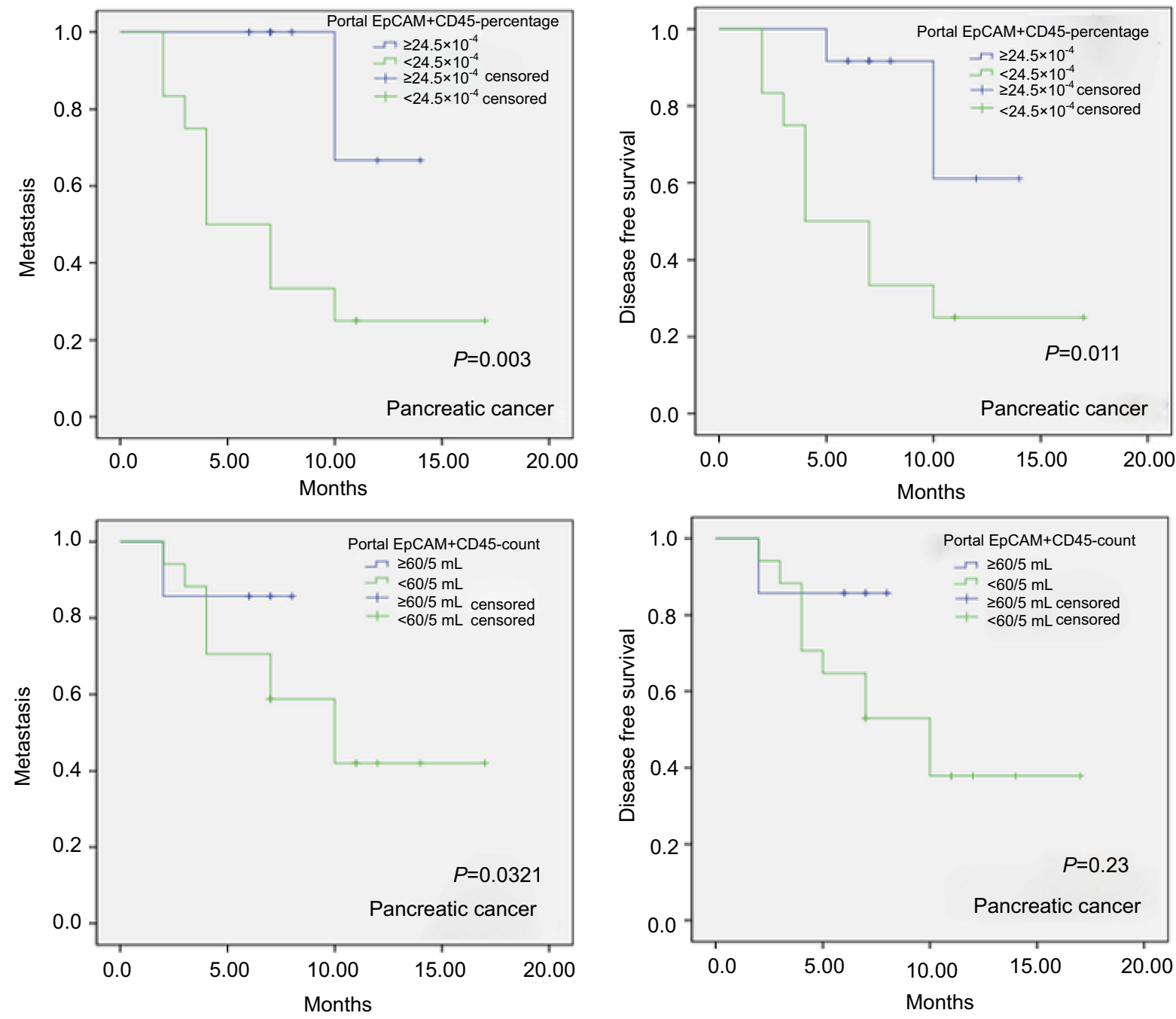

Figure 3 Kaplan-Meier curve of metastasis- and disease-free survival in pancreatic cancer based on a portal EpCAM+CD45- percentage cutoff of $\geq 24.5 \times 10^{-4}$ vs $<24.5 \times 10^{-4}$ and based on a portal EpCAM+CD45- count cutoff of $\geq 60 / 5 \mathrm{~mL}$ vs $<60 / 5 \mathrm{~mL}$.

EpCAM+CD45- region showed significantly different profiles compared to WBCs.

It is surprising that among CTCs that originate from a single patient, each one has a strikingly different copy number profile. Given the intratumoral heterogeneity of pancreatic cancer, ${ }^{27,28}$ it can be expected that this heterogeneity would be reflected in CTCs, as suggested by Lapin and colleagues. ${ }^{29}$ Additional analysis showed that the profile of confirmed CTCs in PC patients was similar to that of FCM-isolated EpCAM+CD45- cells and that their CNV patterns are significantly different from those of WBCs. These results confirm the reliability of FCM for CTC detection and isolation.

In our study, portal venous blood from 39 patients was sampled with a 23-gauge needle during surgery, and no immediate or delayed complications, including hematoma formation or gastrointestinal bleeding, were observed. In a previous study, blood samples were obtained during surgery by direct puncture using a 21-gauge needle under direct visualization, and one patient required suturing with 6-0 Prolene to stop the bleeding. ${ }^{13}$ This finding indicates that using a finer needle for portal vein sampling during surgery may be safer, especially for the typically older cancer patients who may have developed vascular disease.

Moreover, we found that CTCs can be detected at a higher rate and a higher count in portal than in peripheral venous blood in patients with periampullary tumors and PC, which agrees with previous study results. $^{12,13,30}$ Similarly, the number of EpCAM+CD45cells in portal venous blood was higher than that in peripheral venous blood. Although the enumeration of CTCs in the peripheral blood has been shown to be an 


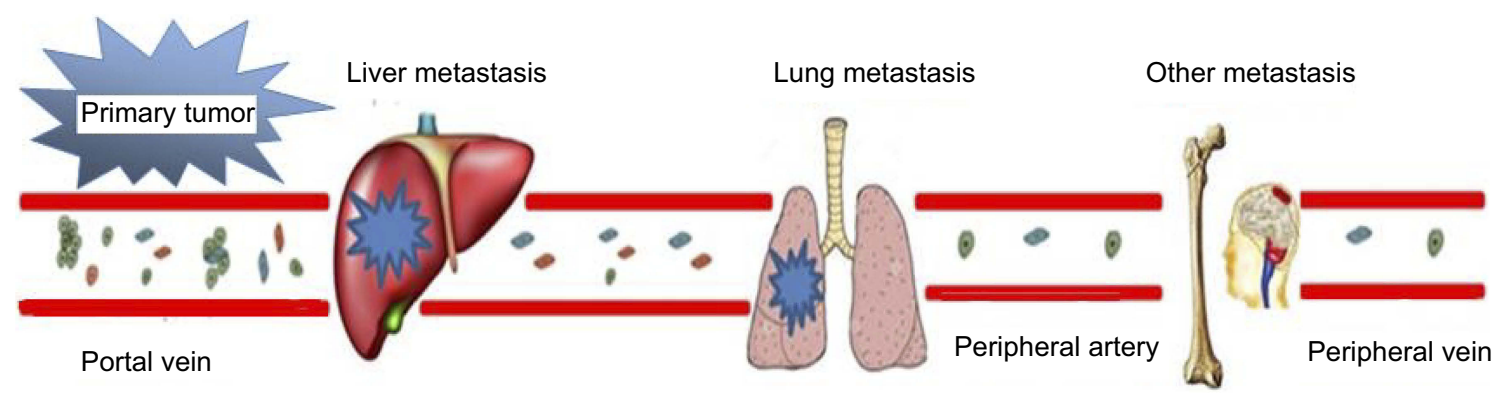

Figure 4 CTCs in the blood of patients with pancreatic or periampullary tumors and relationship with tumor metastasis. Tumor cells from the primary tumor first enter the portal vein in the form of a single cell or small emboli and easily migrate to the liver, causing metastasis; a smaller number of CTCs can migrate to the lung, bone or brain and cause metastatic cancer. Only a few tumor cells appear in the peripheral blood.

informative biomarker for prognostic evaluation in PC patients, ${ }^{11}$ the detection sensitivity is poor due to the low CTC concentration. In addition, postoperative PC primarily metastasizes to the liver, whereas metastasis to sites such as the lungs or peripheral veins is rare. Thus, detection of peripheral blood CTCs is ineffective, and its clinical application is limited. In contrast, liver metastasis is more directly correlated with the presence of CTCs in portal venous blood (Figure 4). Therefore, portal venous blood CTC detection may be more sensitive and specific than detection using peripheral venous blood for the prediction of postoperative metastases in pancreatic or periampullary tumors. Moreover, CTC detection in portal venous blood is a significant predictor of metastasis versus that in systemic venous blood could be explain by less viability of CTC after they pass a heart and the lung capillary system, because this cell traveling inevitably associated with hypoxia, nutrient deprivation, and shear stress. ${ }^{31-33}$

Our study also confirms that CTC-related FCM parameters, especially the portal EpCAM+CD45- percentage, are effective indicators of metastasis and DFS after curative resection in patients with periampullary tumors or PC. However, multivariate analysis was not performed for highly correlated FCM parameters. Among the FCM parameters, only the portal EpCAM $+\mathrm{CD} 45$ - count was not identified as a predictor of metastasis, which may be due to the instability of the blood flow in the portal vein during surgery. CA242, the clinical value of which has been widely proven in cancers originating from the pancreatic and biliary tracts, has also been found to be meaningful for prediction of DFS and metastasis. ${ }^{34,35}$ Similarly, the portal EpCAM + CD45- percentage of PC patients was significantly associated with the risk of developing metastases and
DFS after surgery. The failure of the portal EpCAM $+\mathrm{CD} 45$ - count to be useful in prognostic prediction may be due to the instability of blood flow during surgery. Thus, FCM offers a significant advantage, as it can measure the percentage of CTCs, which is unaffected by variations in blood flow and count and thus provides a reliable and stable indicator. Such an advantage is especially pertinent for CTCs detected in portal venous blood, which are close to the operative region and have a much greater possibility of being disturbed by alterations in blood flow due to bleeding, fluid infusion or local pressure during surgery. Other platforms such as the CellSearch system, microfluidic capture or size-based filtering can provide the CTC count but not the percentage and are therefore not suitable for portal CTC detection.

Although our study has a relatively small sample size, our results showed that the CTCs detected by FCM in portal venous blood are of significant value for the prediction of postoperative metastasis for pancreatic or periampullary tumors. As this is a major finding in this study and our results are statistically significant, the sample size is probably adequate. Moreover, there are several strengths worth emphasizing. First, given that diagnosis of all pancreatic or periampullary tumors, especially PC, was confirmed pathologically and clinically, the present study may have avoided ascertainment bias because of misdiagnosis. Second, the blood collection procedure and specimens were of high quality, and patients who underwent preoperative chemotherapy were excluded from the study.

In conclusion, we analyzed CTC counts in 39 patients with periampullary or pancreatic tumors by collecting blood during surgery. The results indicate that CTC detection in portal venous blood by FCM may be a significant predictor of metastasis and DFS after surgery. Moreover, 
FCM may be a reliable and appropriate CTC detection and isolation platform for clinical settings, particularly for the intraoperative detection of CTCs in portal venous blood.

\section{Abbreviations}

PC, pancreatic cancer; PDAC, pancreatic duct adenocarcinoma; CTC, circulating tumor cell; FCM, flow cytometry; EpCAM, epithelial cell adhesion molecule; CNV, copy number variation; AJCC, American Joint Committee on Cancer; BMCs, blood mononuclear cells; DFS, disease-free survival; OS, overall survival; Cis, confidence intervals.

\section{Acknowledgments}

This study was supported by a grant from the Capital Characteristic Clinical Application Research and Achievement Promotion Project (No. Z171100001017121), the Doctoral Venture Capital Fund of Henan Provincial People's Hospital (No. ZC20180077), the Special Project of Henan Provincial Key Research, Development and Promotion (Science and Technology) (No. 192102310119). We acknowledge the assistance of Dr. Li Yanfang, Yang Yanlin, Chen $\mathrm{Li}$ and $\mathrm{Yu}$ Xiaotong (Medical Research Center, Peking University Third Hospital) with the blood sample processing techniques. We also acknowledge the assistance of Dr. Tao Liyuan (Research Center of Clinical Epidemiology, Peking University Third Hospital) with the statistical analysis. Finally, we extend a special thanks to all patients who provided specimens.

\section{Author contributions}

Dianrong Xiu and Lianyuan Tao conceived and designed this study. Lianyuan Tao and Li Su performed the flow cytometry analysis. Lianyuan Tao processed the samples and performed the laser confocal scanning microscopy. Lianyuan Tao, Shiping Bo, Yunyun Niu and Sijia Lu performed the single-cell sequencing. Dianrong Xiu, Chunhui Yuan and Zhaolai Ma performed the statistical analysis and data interpretation. Lianyuan Tao and Lingfu Zhang collected the clinical data and samples. Dianrong Xiu and Lianyuan Tao wrote the manuscript. All authors contributed to data analysis, drafting or revising the article, gave final approval of the version to be published, and agree to be accountable for all aspects of the work.

\section{Disclosure}

The authors report no conflicts of interest in this work.

\section{References}

1. Dimastromatteo J, Houghton JL, Lewis JS, Kelly KA. Challenges of pancreatic cancer. Cancer J. 2015;21:188-193. doi:10.1097/ PPO.000000000000109

2. Hidalgo M. Pancreatic cancer. $N$ Engl J Med. 2010;362:1605-1617. doi:10.1056/NEJMra0901557

3. Zhang Q, Zeng L, Chen Y, et al. Pancreatic cancer epidemiology, detection, and management. Gastroenterol Res Pract. 2016;2016:8962321. doi:10.1155/2016/8962321

4. Long J, Luo GP, Xiao ZW, et al. Cancer statistics: current diagnosis and treatment of pancreatic cancer in Shanghai, China. Cancer Lett. 2014;346:273-277. doi:10.1016/j.canlet.2014.01.004

5. Chen SC, Shyr YM, Wang SE. Longterm survival after pancreaticoduodenectomy for periampullary adenocarcinomas. HPB (Oxford). 2013;15:951-957. doi:10.1111/hpb.12071

6. Riall TS, Cameron JL, Lillemoe KD, et al. Resected periampullary adenocarcinoma: 5-year survivors and their 6- to 10-year follow-up. Surgery. 2006;140:764-772. doi:10.1016/j.surg.2006.04.006

7. Tao L, Zhang L, Peng Y, et al. Neutrophils assist the metastasis of circulating tumor cells in pancreatic ductal adenocarcinoma: a new hypothesis and a new predictor for distant metastasis. Medicine (Baltimore). 2016;95:e4932. doi:10.1097/MD.0000000000004864

8. Erdmann JI, Morak MJ, Duivenvoorden HJ, et al. Long-term survival after resection for non-pancreatic periampullary cancer followed by adjuvant intra-arterial chemotherapy and concomitant radiotherapy. HPB (Oxford). 2015;17:573-579. doi:10.1111/hpb.12401

9. Chen XX, Bai F. Single-cell analyses of circulating tumor cells. Cancer Biol Med. 2015;12:184-192. doi:10.7497/j.issn.2095-3941.2015.0056

10. Joosse SA, Gorges TM, Pantel K. Biology, detection, and clinical implications of circulating tumor cells. EMBO Mol Med. 2015;7:111. doi: $10.15252 / \mathrm{emmm} .201303698$

11. Han L, Chen W, Zhao Q. Prognostic value of circulating tumor cells in patients with pancreatic cancer: a meta-analysis. Tumour Biol. 2014;35:2473-2480. doi:10.1007/s13277-013-1327-5

12. Bissolati M, Sandri MT, Burtulo G, Zorzino L, Balzano G, Braga M. Portal vein-circulating tumor cells predict liver metastases in patients with resectable pancreatic cancer. Tumour Biol. 2015;36:991-996. doi:10.1007/s13277-014-2716-0

13. Tien YW, Kuo HC, Ho BI, et al. A high circulating tumor cell count in portal vein predicts liver metastasis from periampullary or pancreatic cancer: a high portal venous CTC count predicts liver metastases. Medicine (Baltimore). 2016;95:e3407. doi:10.1097/ MD.0000000000004864

14. Liu X, Li C, Li J, et al. Detection of CTCs in portal vein was associated with intrahepatic metastases and prognosis in patients with advanced pancreatic cancer. $J$ Cancer. 2018;9:2038-2045. doi:10.7150/jca.23989

15. Hugenschmidt $\mathrm{H}$, Labori KJ, Brunborg $\mathrm{C}$, et al. Circulating tumor cells are an independent predictor of shorter survival in patients undergoing resection for pancreatic and periampullary adenocarcinoma. Ann Surg. 2018. doi: $10.1097 /$ SLA.0000000000003035

16. Vilhav C, Engstrom C, Naredi P, et al. Fractional uptake of circulating tumor cells into liver-lung compartments during curative resection of periampullary cancer. Oncol Lett. 2018;16:6331-6338. doi:10.3892/ol.2018.9435

17. Adams DL, Stefansson S, Haudenschild C, et al. Cytometric characterization of circulating tumor cells captured by microfiltration and their correlation to the cellSearch((R)) CTC test. Cytometry A. 2015;87:137-144. doi:10.1002/cyto.a.22613

18. Dong Y, Skelley AM, Merdek KD, et al. Microfluidics and circulating tumor cells. J Mol Diagn. 2013;15:149-157. doi:10.1016/j. jmoldx.2012.09.004

19. Khoja L, Backen A, Sloane R, et al. A pilot study to explore circulating tumour cells in pancreatic cancer as a novel biomarker. Br J Cancer. 2012;106:508-516. doi:10.1038/bjc.2011.545 
20. Lu Y, Liang $\mathrm{H}, \mathrm{Yu} \mathrm{T}$, et al. Isolation and characterization of living circulating tumor cells in patients by immunomagnetic negative enrichment coupled with flow cytometry. Cancer. 2015;121:30363045. doi: $10.1002 /$ cncr. 29444

21. Edge SB, Compton CC. The American joint committee on cancer: the 7th edition of the AJCC cancer staging manual and the future of TNM. Ann Surg Oncol. 2010;17:1471-1474. doi:10.1245/s10434-010-0985-4

22. Pinto LA, Trivett MT, Wallace D, et al. Fixation and cryopreservation of whole blood and isolated mononuclear cells: influence of different procedures on lymphocyte subset analysis by flow cytometry. Cytometry B Clin Cytom. 2005;63:47-55. doi:10.1002/cyto.b.20038

23. Zong C, Lu S, Chapman AR, Xie XS. Genome-wide detection of single-nucleotide and copy-number variations of a single human cell. Science. 2012;338:1622-1626. doi:10.1126/science.1229164

24. Huang L, Ma F, Chapman A, Lu S, Xie XS. Single-cell whole-genome amplification and sequencing: methodology and applications. Annu Rev Genomics Hum Genet. 2015;16:79-102. doi:10.1146/annurev-genom-090413-025352

25. Ni X, Zhuo M, Su Z, et al. Reproducible copy number variation patterns among single circulating tumor cells of lung cancer patients. Proc Natl Acad Sci U S A. 2013;110:21083-21088. doi:10.1073/ pnas.1320659110

26. Yuan J, Zhou J, Dong Z, et al. Pretreatment serum VEGF is associated with clinical response and overall survival in advanced melanoma patients treated with ipilimumab. Cancer Immunol Res. 2014;2:127-132. doi:10.1158/2326-6066.CIR-13-0163

27. Kim MS, Zhong Y, Yachida S, et al. Heterogeneity of pancreatic cancer metastases in a single patient revealed by quantitative proteomics. Mol Cell Proteomics. 2014;13:2803-2811. doi:10.1074/ mcp.M114.038547
28. Cros J, Raffenne J, Couvelard A, et al. Tumor heterogeneity in pancreatic adenocarcinoma. Pathobiology. 2018;85:64-71. doi: $10.1159 / 000477773$

29. Lapin M, Tjensvoll K, Oltedal S, et al. Single-cell mRNA profiling reveals transcriptional heterogeneity among pancreatic circulating tumour cells. BMC Cancer. 2017;17:390. doi:10.1186/s12885-0173385-3

30. Catenacci DV, Chapman CG, Xu P, et al. Acquisition of portal venous circulating tumor cells from patients with pancreaticobiliary cancers by endoscopic ultrasound. Gastroenterology. 2015;149:1794-1803 e4. doi:10.1053/j.gastro.2015.08.050

31. Hong Y, Fang F, Zhang Q. Circulating tumor cell clusters: what we know and what we expect (Review). Int J Oncol. 2016;49:22062216. doi:10.3892/ijo.2016.3747

32. Labelle M, Hynes RO. The initial hours of metastasis: the importance of cooperative host-tumor cell interactions during hematogenous dissemination. Cancer Discov. 2012;2:1091-1099. doi:10.1158/ 2159-8290.CD-12-0329

33. Patel LR, Camacho DF, Shiozawa Y, Pienta KJ, Taichman RS. Mechanisms of cancer cell metastasis to the bone: a multistep process. Future Oncol. 2011;7:1285-1297. doi:10.2217/fon.11. 112

34. Ni XG, Bai XF, Mao YL, et al. The clinical value of serum CEA, CA19-9, and CA242 in the diagnosis and prognosis of pancreatic cancer. Eur J Surg Oncol. 2005;31:164-169. doi:10.1016/j. ejso.2004.09.007

35. Tao LY, Cai L, He XD, Liu W, Qu Q. Comparison of serum tumor markers for intrahepatic cholangiocarcinoma and hepatocellular carcinoma. Am Surg. 2010;76:1210-1213. 


\section{Supplementary materials}
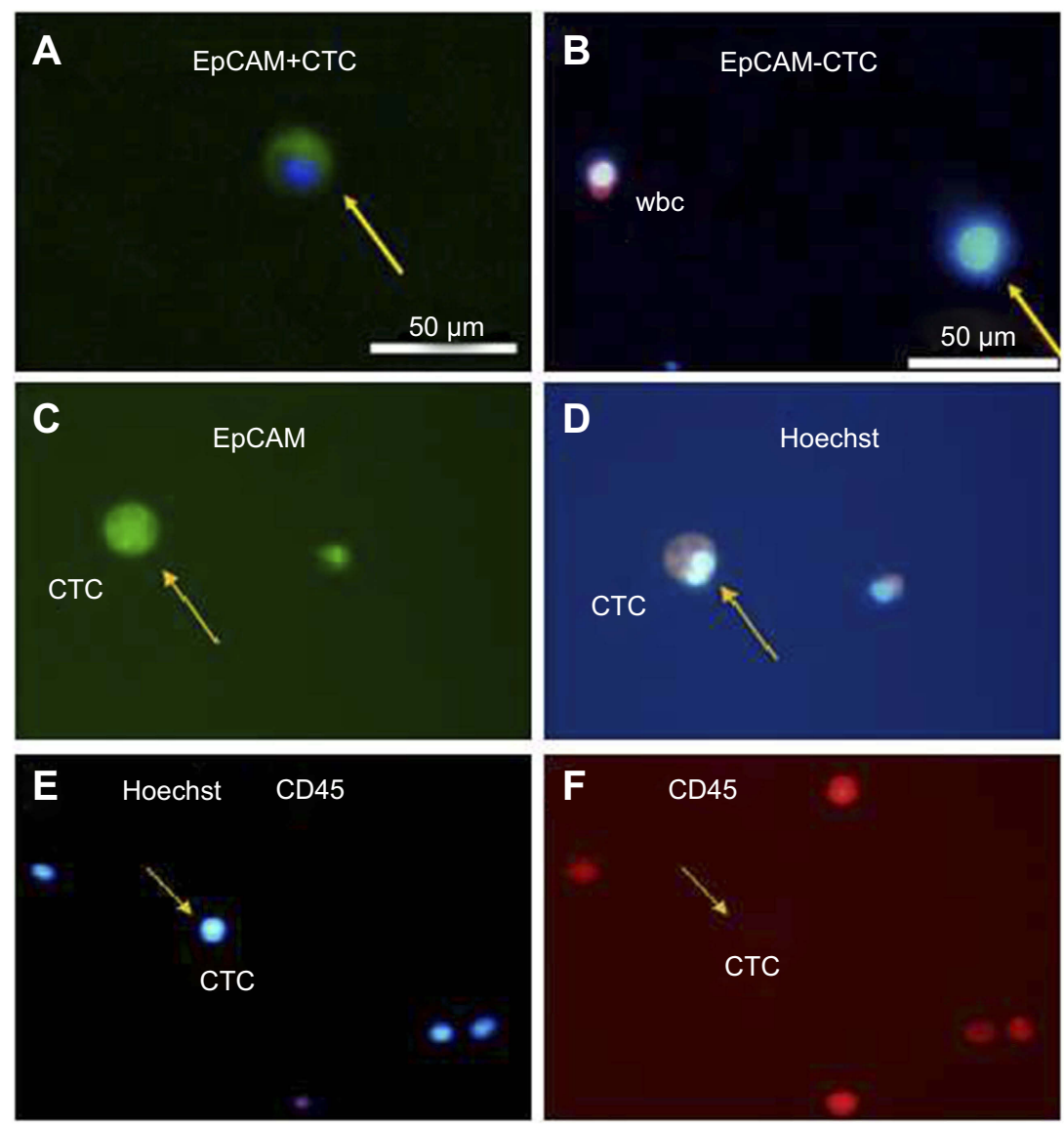

Figure SI (A) EpCAM+ cells meeting the morphological criteria were identified as CTCs (Hoechst: blue; EpCAM-FITC: green); (B) CD45- cells meeting the morphological criteria were identified as CTCs (Hoechst: blue; CD45-PE: red). 

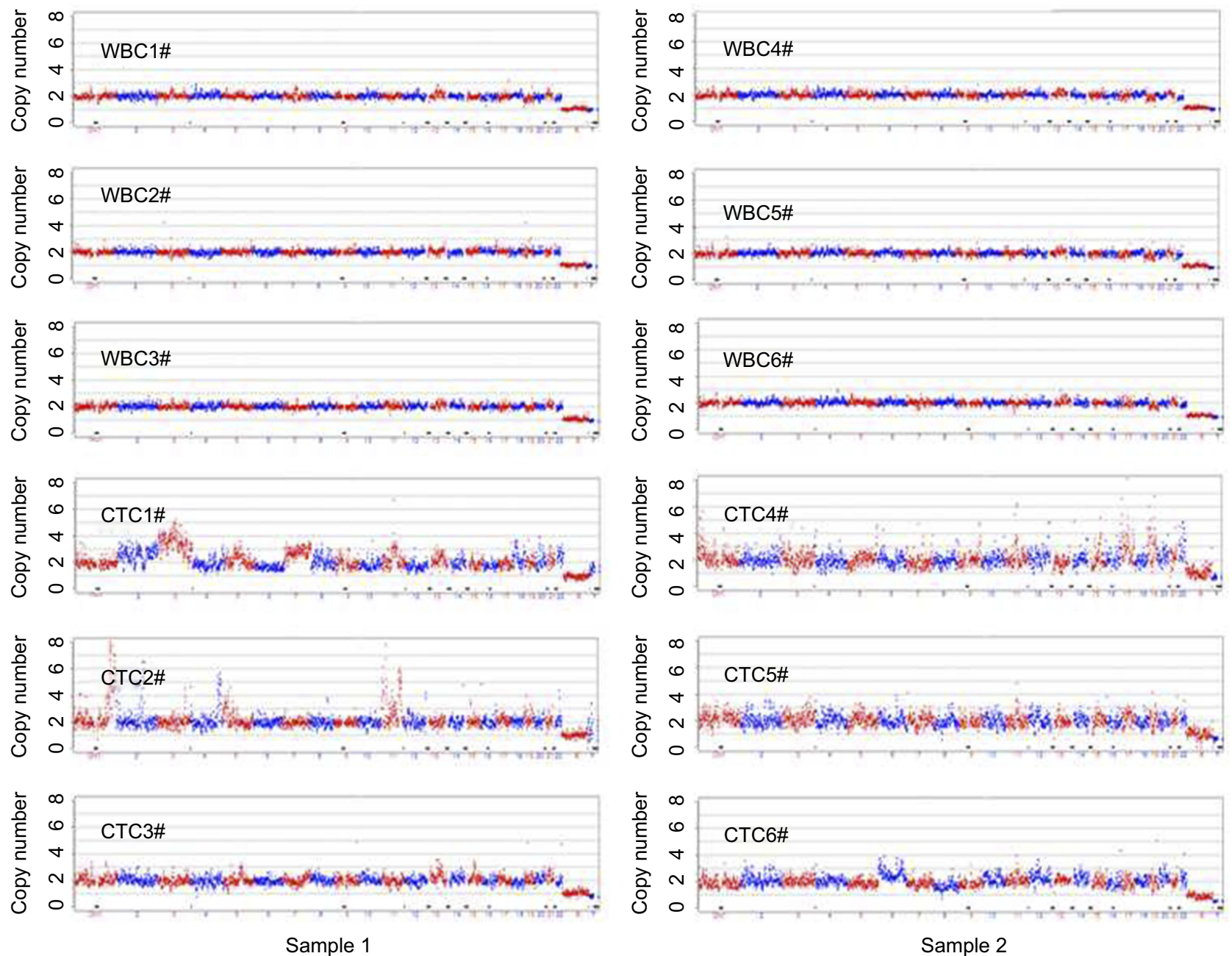

Figure 52 CNVs in CTCs and WBCs from two pancreatic cancer patients (sample I and sample 2). The CNV patterns of CTCs in both sample I and sample 2 are significantly different from those of WBCs. The copy numbers are segmented (blue and red lines). 

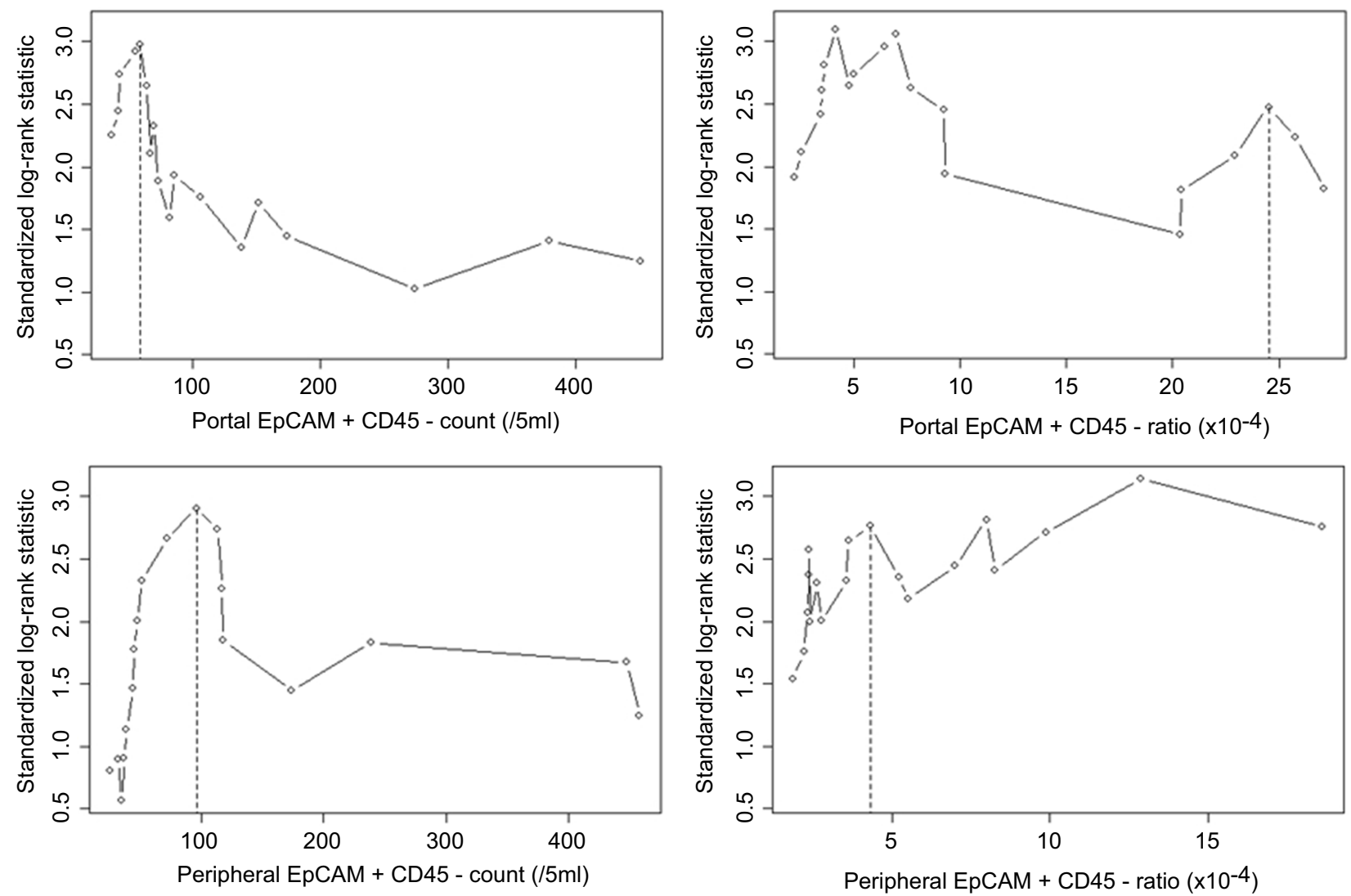

Figure S3 Cutoff point of FCM parameters defined using maximally selected log-rank statistics for disease-free survival in periampullary and pancreatic tumor patients. Maximally selected log-rank statistics were used to define possible cutoff points for FCM parameters. Four FCM parameters were found to be associated with DFS in all patients (portal EpCAM+CD45- count, $P=0.024$ for a cutoff of $59 / 5 \mathrm{~mL}$; portal EpCAM+CD45- percentage, $P=0.018$ for a cutoff of $4.2 \times 10^{-4}$; peripheral EpCAM+CD45count, $P=0.03$ for a cutoff of $96 / 5 \mathrm{~mL}$; and peripheral EPCAM+CD45- percentage $P=0.015$ for a cutoff of $12.86 \times 10^{-4}$ ). The cutoff points were then adjusted according to participant distribution into two groups for all patients $\left(60 / 5 \mathrm{~mL}, 24.5 \times 10^{-4}\right)$ as well as for pancreatic cancer patients $\left(97 / 5 \mathrm{~mL}, 4.4 \times 10^{-4}\right)$. 


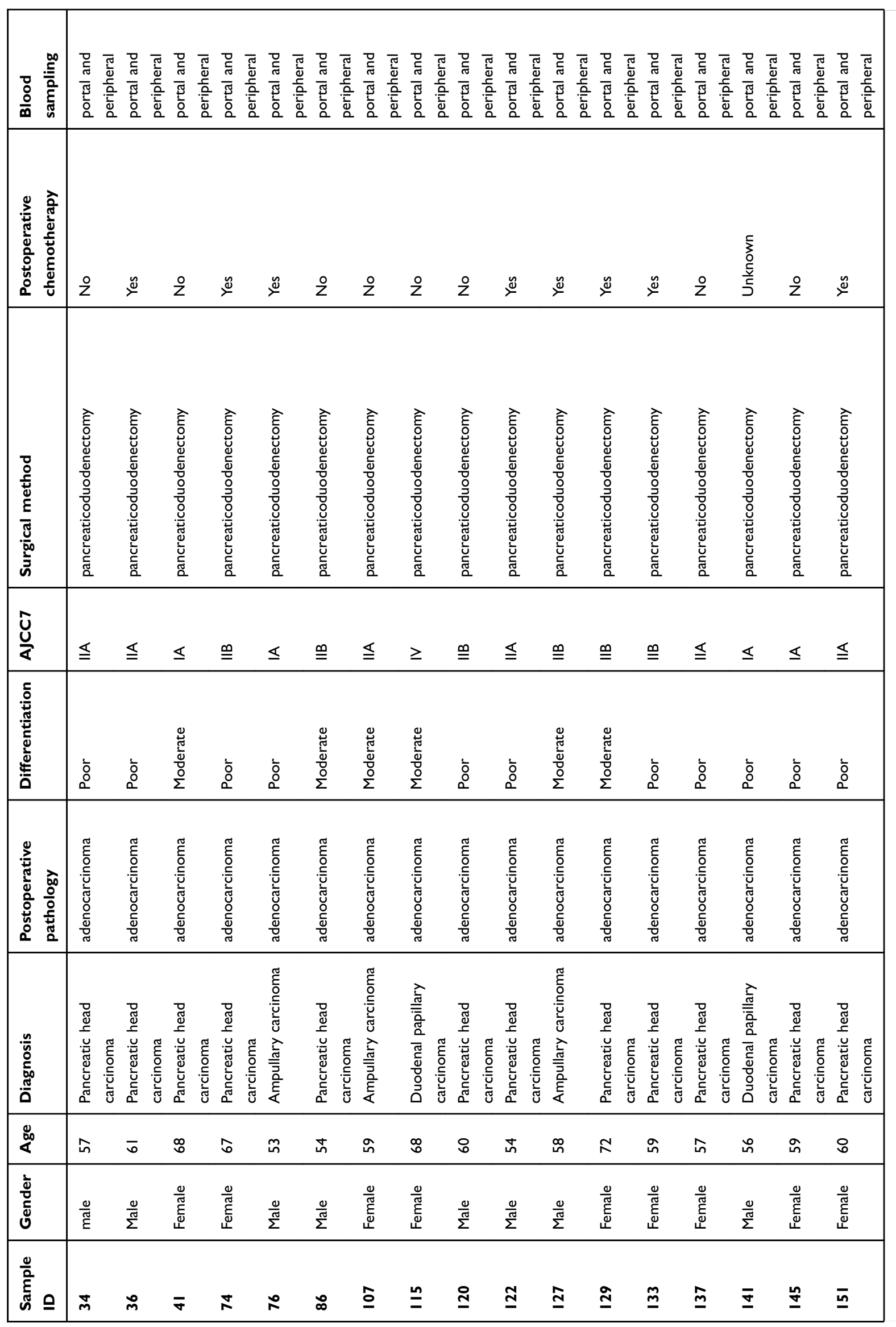




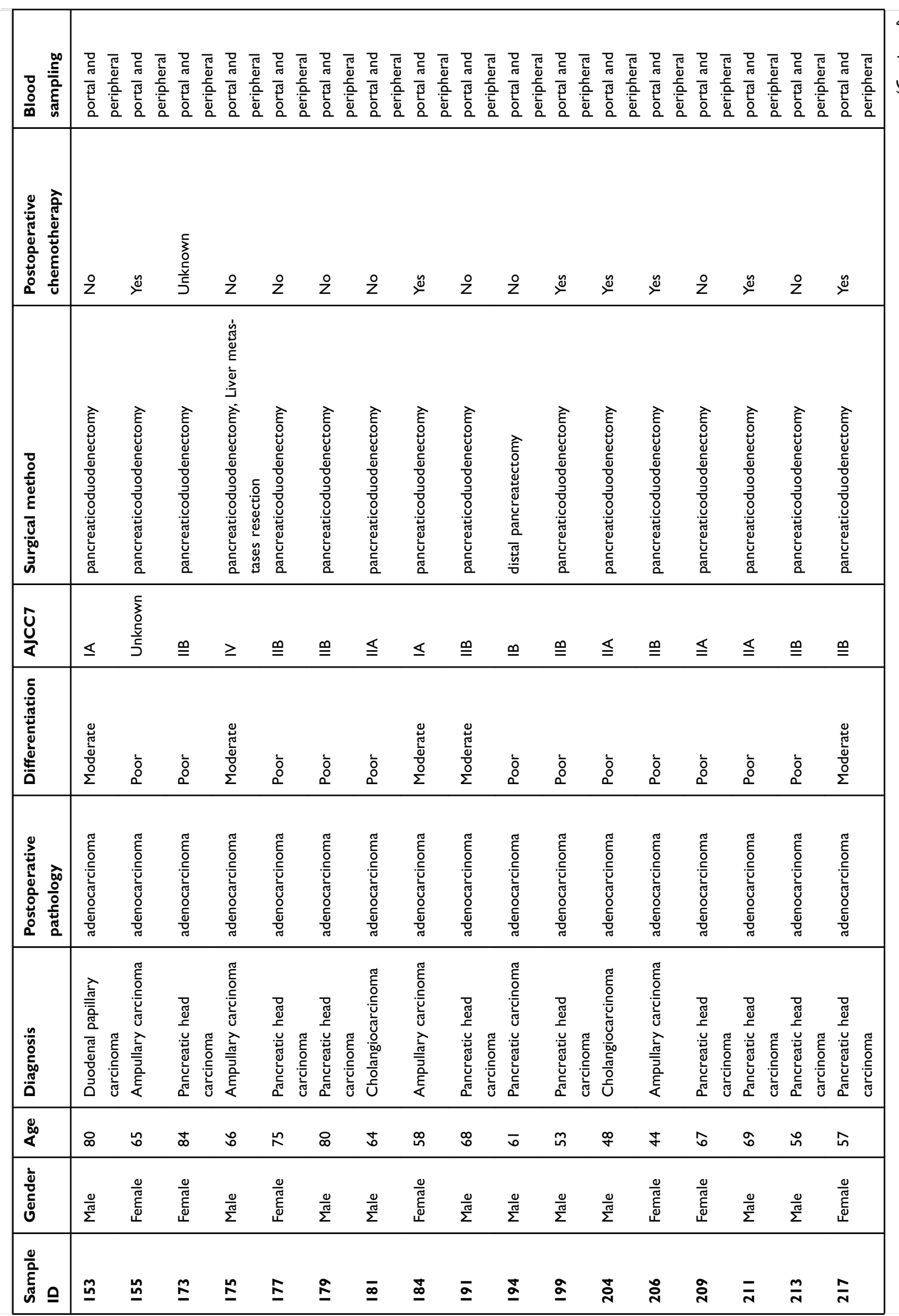




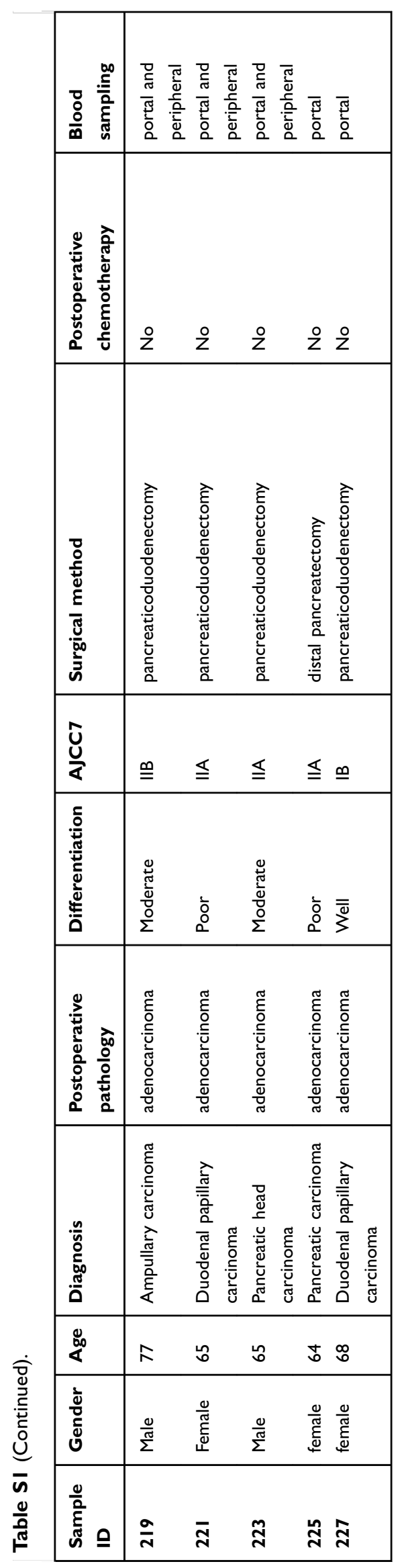


Table S2 Detection Results of CVN of CTCs based on single-cell sequencing

\begin{tabular}{|l|l|l|}
\hline CTCs & Detection Results & Amplification \\
\hline EpCAM + CD45 - I\# & $46, X N,-2 p,-2 q,-6 p$ & Normal \\
EpCAM + CD45 - 2\# & $46, X N$ cnv normal & Normal \\
EpCAM + CD45 - 3\# & $46, X N,+3 q,+9 q,+12 q,+15 q$ & Normal \\
EpCAM + CD45 - 4\# & $46, X N,+4 q\left(m o s^{*}, 25 \%\right)$ & Normal \\
EpCAM + CD45 - 5\# & $46, X N,+7 q\left(m o s^{*}, 250 \%\right)$ & Normal \\
EpCAM + CD45 + I\# & $46, X N$ cnv normal & Normal \\
EpCAM + CD45 + 2\# & $46, X N$ cnv normal & Normal \\
EpCAM + CD45 + 3\# & Amplification abnormal & Abnormal \\
EpCAM + CD45 + 4\# & Multiple chromosomal abnormalities;+lp,+lq,+2p,+6p,+lop. & Abnormal \\
\hline
\end{tabular}

Notes: According to the An International System for Human Cytogenetic Nomenclature $(2013)^{1}+$ indicates a duplication; - indicates a deletion; mos* indicates suspicious mosaicism; $p$ indicates the short arm of a chromosome and $q$ indicates the long arm of a chromosome.

\section{Reference}

1. Shaffer LG, McGowan-Jordan J, Schmid M, editors. An International System for Human Cytogenetic Nomenclature (2013). Basel: Karger Publishers; 2013.

\section{Publish your work in this journal}

Cancer Management and Research is an international, peer-reviewed open access journal focusing on cancer research and the optimal use of preventative and integrated treatment interventions to achieve improved outcomes, enhanced survival and quality of life for the cancer patient.
The manuscript management system is completely online and includes a very quick and fair peer-review system, which is all easy to use. Visit http://www.dovepress.com/testimonials.php to read real quotes from published authors. 